\title{
A phantom study comparing technical image quality of five breast tomosynthesis systems
}

\section{Sundell, Veli-Matti}

2019-07

Sundell , V-M , Jousi , M , Hukkinen, K, Blanco , R , Mäkelä , T \& Kaasalainen , T 2019 , ' A phantom study comparing technical image quality of five breast tomosynthesis systems ' , Physica Medica , vol. 63 , pp. 122-130 . https://doi.org/10.1016/j.ejmp.2019.06.004

http://hdl.handle.net/10138/316118

https://doi.org/10.1016/j.ejmp.2019.06.004

cc_by_nc_nd

acceptedVersion

Downloaded from Helda, University of Helsinki institutional repository.

This is an electronic reprint of the original article.

This reprint may differ from the original in pagination and typographic detail.

Please cite the original version. 


\section{A phantom study comparing technical image quality of five breast tomosynthesis sys-}

\section{tems}

Veli-Matti Sundell ${ }^{1,2}$, Mikko Jousi ${ }^{3}$, Katja Hukkinen ${ }^{1}$, Roberto Blanco ${ }^{4}$, Teemu Mäkelä ${ }^{1,2}$, Touko Kaasalainen ${ }^{1,2}$ 1 HUS Medical Imaging Center, Radiology, University of Helsinki and Helsinki University Hospital, Finland

2 Department of Physics, University of Helsinki, Finland

3 Päijät-Hämeen Sosiaali- ja Terveysyhtymä, Central Hospital, Radiology, Finland

4 Medical Imaging Centre of Southwest Finland, Turku University Hospital

Corresponding author:

Veli-Matti Sundell

HUS Medical Imaging Center, Radiology, University of Helsinki and Helsinki University Hospital P.O. Box 340 (Haartmaninkatu 4),

00290 Helsinki, Finland

veli-matti.sundell@helsinki.fi

Conflict of interest: Mr. Mikko Jousi is a medical advisor for Planmed Oy. 


\title{
A phantom study comparing technical image quality of five breast tomosynthesis sys- tems
}

\begin{abstract}
Background: Digital breast tomosynthesis (DBT) is a three-dimensional breast imaging method. DBT vendors employ various approaches in both image acquisition and data processing, which may affect image quality and radiation exposure to patients.

Objective: This study aimed to evaluate the performance of five DBT systems: Fujifilm Amulet Innovality (using both a standard mode and high-resolution mode), GE Senographe Essential, Hologic Selenia Dimensions, Planmed Clarity 3D, and Siemens Mammomat Inspiration.

Materials and methods: The performance of each device and imaging technique was evaluated and compared by phantom measurements performed with four quality assurance phantoms. Technical image quality assessments consisted of measuring artefact extent, in-plane resolution, relative noise power spectrum, and geometric accuracy.
\end{abstract}

Results: Artefact spreading varied remarkably between the devices, and the full width at half maximum values of artefact spread functions varied from $3.5 \mathrm{~mm}$ to $10.7 \mathrm{~mm}$. Noticeable in-plane resolution anisotropy, determined using modulation transfer function (MTF) analysis, was typically observed between tube travel direction and chest wall-nipple direction. The $\mathrm{MTF}_{50}$ varied from $1.1 \mathrm{~mm}^{-1}$ to $1.6 \mathrm{~mm}^{-1}$ and from $1.5 \mathrm{~mm}^{-1}$ to $4.1 \mathrm{~mm}^{-1}$ in the tube travel and chest wall-nipple directions, respectively. Moreover, distinctly different noise power spectra were observed between the systems. The geometric accuracy in every system was within $0.5 \%$.

Conclusion: Technical image quality assessments with image quality phantoms revealed remarkable differences in artefact spread, in-plane resolution, and noise properties between the DBT systems and imaging methods.

Keywords: Digital breast tomosynthesis; Image quality; Quality control 


\section{INTRODUCTION}

Digital breast tomosynthesis (DBT) is a novel three-dimensional imaging technique that is used to detect malignancies in breast tissue. Recently, the use of DBT has increased due to higher accuracy compared to conventional mammography in detecting breast-tissue malignancies [1]. By using DBT, the interference from an overlapping fibroglandular breast tissue can be reduced, improving the differentiation between malignant and non-malignant tissue features. The use of DBT in breast cancer screening has been shown to increase cancer detection rate when used together with digital mammography [2-6], and to reduce the need for recalls $[3,5,7,8]$. There are, however, varying results considering DBT's sensitivity for detecting microcalcifications typically seen in breast cancer [913]. This may partly stem from the differences in scanning and image reconstruction techniques [14].

In DBT, several projection images are acquired while rotating the x-ray tube and, in some systems, the detector about the breast. . Exposures are made at predetermined intervals and angles. The angular range and scan geometry vary from one manufacturer to another. The acquired projection images are processed with image reconstruction algorithms, either filtered back projection (FBP) or iterative reconstruction algorithms. Generally, the tissues in the in-focus plane are reproduced sharply while the details of higher and lower image planes become blurred. The image quality is highly dependent on the DBT systems' geometry, image acquisition method, reconstruction algorithm, and scanning parameters.

Each DBT vendor offers unique technical approaches. The main differences between the systems are the ways of controlling the x-ray tube motion (continuous or step-and-shoot), detector motion (stationary, rotating, or syncand-shoot), the angular range of the $\mathrm{x}$-ray tube rotation in a single acquisition $\left(15-50^{\circ}\right)$, image reconstruction (FBP or iterative reconstruction algorithms), and other image post-processing features [15,16]. Additionally, the x-ray tube target material, filter material, detector material, pixel size, and pixel binning vary. Whether and how the differences in the technical implementations affect the detection and characterization of breast cancer is still under research. Until now, a few studies have investigated the performance of different DBT systems [17-23]. These studies and works by Sechopoulos [15] and Mainprize et al. [24] have, for example, indicated that a wide scan angle range would provide improved depth resolution while a narrow range would improve the in-plane resolution. Quality control procedures for DBT systems are under active research. European Reference Organisation for Quality Assured Breast Screening and Diagnostic Services (EUREF) has published quality control protocols for DBT 
systems [16], and European Federation of Organisations for Medical Physics (EFOMP) has recently approved a new Working Group on DBT QA procedures [25].

Due to ethical reasons, it is difficult and impractical to compare the performance of several DBT systems for the same patient. Our phantom study aimed to explore objective and comparable technical image quality in five DBT systems. We examined artefact spread in z-direction (using artefact spread function, ASF), image resolution in $\mathrm{x}$ and y-directions (using modulation transfer function, MTF), relative noise power spectra (NPS), and geometric accuracy. The comparability between different systems requires standardized imaging setups and concise analysis. The novelty of our work is in that no previous studies have compared all of the presented DBT systems.

\section{MATERIALS AND METHODS}

Technical image quality of five clinically used DBT systems were assessed with phantom measurements. The systems investigated were: Fujifilm Amulet Innovality with the software version V7.0.0007 (Fujifilm Corporation, Tokyo, Japan) using two different scanning modes—-high-resolution (HR) and standard (ST); GE Senographe Essential with the software version 1.10.3 (GE Healthcare, Waukesha, WI, USA); Hologic Selenia Dimensions with the software version 1.7.4.5 (Hologic Inc., Bedford, MA, USA); Planmed Clarity 3D with the software version 1.1.1.10 (Planmed, Helsinki, Finland); and Siemens Mammomat Inspiration with the software version VB41ASL65P66 (Siemens Healthcare, Erlangen, Germany). The detector materials, detector element pitches, detector motions, x-ray tube target materials, filter materials, x-ray tube motions, angular ranges, number of projection images per scan, and reconstruction methods are described in table 1.

Table 1 - Technical specifications of the DBT systems used in the study.

\begin{tabular}{l|ccccc} 
System & $\begin{array}{c}\text { Fujifilm Amulet } \\
\text { Innovality }\end{array}$ & $\begin{array}{c}\text { GE Senographe } \\
\text { Essential }\end{array}$ & $\begin{array}{c}\text { Hologic Selenia } \\
\text { Dimensions }\end{array}$ & $\begin{array}{c}\text { Planmed Clarity } \\
\text { 3D }\end{array}$ & $\begin{array}{c}\text { Siemens Mam- } \\
\text { momat Inspira- } \\
\text { tion }\end{array}$ \\
\hline $\begin{array}{l}\text { Detector mate- } \\
\text { rial }\end{array}$ & a-Se & CsI-a-Si & a-Se & CsI-a-Si & a-Se \\
$\begin{array}{l}\text { Detector ele- } \\
\text { ment pitch }(\mu \mathrm{m})\end{array}$ & $68^{1}$ & 100 & 70 & 83 & 85 \\
$\begin{array}{l}\text { Reconstructed } \\
\text { image pixel size } \\
(\mu \mathrm{m})\end{array}$ & $\begin{array}{c}132.9 \ldots 146.5 \\
(\mathrm{ST})^{2}\end{array}$ & 100 & $103.0 \ldots 112.8^{2}$ & 95 & 85 \\
$88.5 \ldots 97.7$ & & & & \\
$(\mathrm{HR})^{2}$ & & & &
\end{tabular}




\begin{tabular}{|c|c|c|c|c|c|}
\hline Detector motion & Static & Static & Rotating & $\begin{array}{l}\text { Rotating during } \\
\text { exposure (Sync- } \\
\text { and-shoot) }\end{array}$ & Static \\
\hline $\begin{array}{l}\text { X-ray tube tar- } \\
\text { get }\end{array}$ & $\mathrm{W}$ & $\mathrm{Mo} / \mathrm{Rh}$ & $\mathrm{W}$ & W & $\mathrm{W}$ \\
\hline$X$-ray filter & $\mathrm{Al}: 700 \mu \mathrm{m}$ & $\begin{array}{l}\text { Mo: } 30 \mu \mathrm{m} \\
\text { Rh: } 25 \mu \mathrm{m}\end{array}$ & $\mathrm{Al}: 700 \mu \mathrm{m}$ & $\begin{array}{l}\text { Rh: } 75 \mu \mathrm{m} \\
\text { Ag: } 60 \mu \mathrm{m}\end{array}$ & $\mathrm{Rh}: 50 \mu \mathrm{m}$ \\
\hline $\begin{array}{l}\text { X-ray tube mo- } \\
\text { tion }\end{array}$ & Continuous & Step-and-shoot & Continuous & Continuous & Continuous \\
\hline $\begin{array}{l}\text { Angular range } \\
\left(^{\circ}\right)\end{array}$ & $\begin{array}{l}15(\mathrm{ST}) \\
40(\mathrm{HR})\end{array}$ & 25 & 15 & 30 & 50 \\
\hline $\begin{array}{l}\text { Number of pro- } \\
\text { jection images }\end{array}$ & 15 & 9 & 15 & 15 & 25 \\
\hline $\begin{array}{l}\text { Reconstruction } \\
\text { method }\end{array}$ & FBP & Iterative & FBP & Iterative & FBP \\
\hline Detector element & e hexagonally & ped. & & & \\
\hline
\end{tabular}

Dose display accuracies were measured using a RaySafe Xi R/F dosimeter (Unfors RaySafe AB, Billdal, Sweden). A 45-mm-thick polymethyl methacrylate (PMMA) phantom, simulating a "standard" $50 \mathrm{~mm}$ breast with $50 \%$ of adipose tissue and 50\% of glandular tissue, was imaged with automatic exposure control. The resulting imaging parameters $(\mathrm{kVp}, \mathrm{mAs}$, and filter/anode) were then applied for the dose measurements performed in air. After verifying that the DBT systems' dose display accuracies were within $\pm 12 \%$, we explored system performances using custom-made image quality phantoms (Figure 1). We measured the ASF in z-direction, in-plane resolutions in tube travel direction (x-direction) and chest wall-nipple direction (y-direction), relative noise power spectrum, and geometric accuracy of each system. The phantom configurations, phantom thicknesses, and phantom positioning were identical among the systems. Each scan was performed with a 5 daN compression force. Moreover, we used processed images for evaluating image quality. The reconstructed slices were parallel to the detector plane and the slice spacing was set to $1 \mathrm{~mm}$. Most of the devices showed pixel size information of each reconstructed slice in the DICOM (Digital Imaging and Communications in Medicine) header. However, the DICOM metadata of the Hologic DBT system showed only one pixel size value for the whole image stack. This was determined to be for the plane in the middle of the imaged object. The pixel size for other image slices was calculated as 


$$
\text { pixel size }=\frac{\text { reconstructed resolution } \cdot \text { source-to-slice distance }}{\text { source-to-image distance }},
$$

where the source-to-image distance was the distance between the x-ray source and the phantom middle. Automatic exposure control was used in the measurements. Mean glandular doses are given in Appendix 1.

\subsection{Artefact extent in z-direction}

Small structures can manifest themselves in multiple adjacent slices. This effect is enhanced due to incomplete angular sampling. To measure the artefact extent, we scanned four bead phantoms and determined the ASF from the reconstructed images. The PMMA phantoms were $6 \mathrm{~cm}$ thick and contained 31 steel beads that each had a diameter of $1 \mathrm{~mm}$. A phantom image and bead indexing used in the study are shown in figure 2. The ASF measurements were performed at four different depths (all beads positioned to $12 \mathrm{~mm}, 24 \mathrm{~mm}, 36 \mathrm{~mm}$, and $48 \mathrm{~mm}$ from the bottom of the phantom). The investigation was limited to the 15 most central beads that were in the useful field of view of all the DBT systems.

To analyze images, we first made a background gradient subtraction by fitting a first-order two-dimensional polynomial function to the image and subtracting it from the original image. We then calculated individual raw ASFs for the 15 beads as

$$
A S F(z)=\frac{P V_{\max }(z)-P V_{b g}(z)}{P V_{\max }\left(z_{0}\right)-P V_{b g}\left(z_{0}\right)} \cdot \frac{S D_{b g}\left(z_{0}\right)}{S D_{b g}(z)}
$$

where the $z$ represents the $\mathrm{z}$-coordinate of a certain slice, $z 0$ is the in-focus plane of the bead, $P V_{\max }$ is the maximum pixel value of the bead or artefact in that plane, $P V_{b g}$ is the mean pixel value of the background, and $S D_{b g}$ is the corresponding standard deviation of the background [21, 26, 27]. The background area was selected to be $100 \mathrm{x}$ 100 pixels and the area to calculate the maximum values of artefacts $150 \times 30$ pixels.

After calculating the raw ASF, we subtracted the baseline and normalized the ASF with its maximum value. Furthermore, we calculated the full width at half maximum (FWHM) value for each ASF and determined the mean ASF-FWHM values for each DBT system at four different bead depths. ASF describes the extent of high contrast object intensity intrusion from the actual location to adjacent slices. Therefore, a small ASF-FWHM is desired and indicates well localized details without extensive spreading in the z-direction. 


\subsection{In-plane resolution}

To determine in-plane resolution (i.e. "blurring" in the $\mathrm{x}$ - and y-directions), we used a $25 \mu \mathrm{m}$ tungsten wire positioned $4 \mathrm{~cm}$ above the breast support table and in between two PMMA blocks. Total phantom thickness was $5 \mathrm{~cm}$. The tungsten wire was first aligned in the $y$ - and then in the $\mathrm{x}$-direction with an approximately $3^{\circ}$ tilt to measure the directional line spread functions (and MTFs). In the MTF analysis, we first searched for an image plane where the tungsten wire was focused. The angle of the wire was then determined by a linear fit and the image was rotated to the closest axis using bicubic interpolation. An average line spread function was calculated over the wire from an area of 1000 x 50 pixels. This was Fourier transformed to obtain the corresponding MTF. The MTF curves were then normalized to maximum value of one. $\mathrm{MTF}_{\max }$ and $\mathrm{MTF}_{50}$ values were recorded to represent in-plane resolutions in tube travel direction and in chest wall-nipple direction.

\subsection{Image noise}

We evaluated the image noise using relative noise power spectrum. NPS defines how the image noise is distributed in different spatial frequencies. NPS shows how much image noise there is in total and also characterizes the size distribution of the "grainy background clutter" components. Generally, high noise power at high spatial frequencies makes it difficult to distinguish small objects from the background and conversely power at low noise frequencies deteriorates larger image features. The relative NPS values were determined from the same phantom images as used in the in-plane resolution determination. However, the relative NPS values were calculated from an area of 512 x 512 pixels in center area of reconstructed plane that did not contain signal from the tungsten wire. We first fitted a fourth order two-dimensional polynomial to the image and removed it from the original image. We then divided the area of interest to eight overlapping areas each containing 256 x 256 pixels. Finally, we calculated the two-dimensional NPS as

$$
N P S=\frac{1}{n} \sum_{i=1}^{n} \frac{\mid F T_{2 D}\left\{I_{i}\right\}^{2} \Delta x \Delta y}{N \cdot M},
$$

where the $n$ is the number of areas, $I_{i}$ are the pixel values (after polynomial subtraction) of the area $i . N$ and $M$ are the length and width of the area in pixels $(\mathrm{M}=\mathrm{N}=256)$, and $\Delta x$ and $\Delta y$ are the pixel lengths of the reconstructed images in $\mathrm{x}$ - and $\mathrm{y}$ - directions. $F T_{2 D}\{\}$ denotes two-dimensional Fourier transformation. To calculate the one-dimensional NPS in the x- and y-directions, we averaged the two-dimensional NPS over an area around the axes that 
was \pm 7 pixels wide excluding the axes themselves. Finally, the one-dimensional NPS curve was divided by its maximum value to obtain the relative NPS curve.

\subsection{Geometric accuracy}

The geometric accuracy phantom contained two 3-cm-thick PMMA plates and one thin metallic plate with engraved marks. Engraved marks were at a known distance from each other. The plate was imaged at three different heights: beneath $6 \mathrm{~cm}$ of homogeneous PMMA plates (denoted henceforth as height $0 \mathrm{~cm}$ ), in between the PMMA plates $(3 \mathrm{~cm})$, and on top of the PMMA plates $(6 \mathrm{~cm})$. The measured distances between the marked points in the phantom were compared to the known values $(120 \mathrm{~mm}$ and $100 \mathrm{~mm})$. Additionally, the artefacts produced by the objects in the phantom were evaluated visually.

\section{RESULTS}

\subsection{Artefact spread function}

Table 2 shows the mean ( \pm standard deviation) ASF-FWHM values measured for each DBT system at four bead heights. Noticeable differences in the ASF values were observed between the systems. Additionally, a small dependency on the bead depth was also observed. In most systems, ASF-FWHM values decreased when beads were closer to the top position (exception being Fujifilm HR and Planmed systems). The highest ASF-FWHM values were observed for the Planmed system, whereas the Fujifilm DBT system using HR imaging technique produced the lowest ASF values. In addition to FWHM, differing ASF shapes were also observed. This can be seen in figure 3 for the bead number three in the mid-top position.

Table 2 - ASF-FWHM values (mean \pm std) measured for different DBT systems in four different bead heights (measured from the bottom).

\begin{tabular}{l|cccc} 
& \multicolumn{4}{|c}{ ASF-FWHM } \\
DBT system & Bottom (12 $\mathbf{~ m m})$ & Mid-bottom $(\mathbf{2 4} \mathbf{~ m m})$ & Mid-top (36 mm) & Top (48 mm) \\
& {$[\mathbf{m m}]$} & {$[\mathbf{m m}]$} & {$[\mathbf{m m}]$} & {$[\mathbf{m m}]$} \\
\hline Fujifilm HR & $3.5 \pm 0.1$ & $3.6 \pm 0.1$ & $3.5 \pm 0.1$ & $3.5 \pm 0.1$ \\
Fujifilm ST & $9.8 \pm 0.1$ & $9.5 \pm 0.1$ & $9.4 \pm 0.1$ & $9.1 \pm 0.2$ \\
GE & $7.7 \pm 0.3$ & $7.5 \pm 0.3$ & $7.4 \pm 0.1$ & $7.1 \pm 0.1$ \\
Hologic & $9.1 \pm 0.1$ & $9.0 \pm 0.4$ & $8.7 \pm 0.1$ & $8.6 \pm 0.2$ \\
Planmed & $10.3 \pm 1.9$ & $10.2 \pm 2.2$ & $10.7 \pm 1.9$ & $10.6 \pm 1.7$
\end{tabular}




\subsection{In-plane resolution}

Table 3 shows the $\mathrm{MTF}_{\max }$ and $\mathrm{MTF}_{50 \%}$ values in the tube travel and chest wall-nipple directions. The MTF curves shown in figure 4 show noticeable differences in the resolutions between the DBT systems. The resolution was higher in the y-direction than in the $\mathrm{x}$-direction in all systems: the $\mathrm{MTF}_{50}$ values in the tube travel direction were 14-69\% lower compared to the chest wall-nipple direction. The Planmed DBT system had an almost isotropic resolution whereas other DBT systems had highly anisotropic resolution. Moreover, the corresponding shifts in the $\mathrm{MTF}_{\max }$ positions can also be seen.

Table 3 - Spatial frequencies of $\mathrm{MTF}_{\max }$ and $\mathrm{MTF}_{50}$ in the tube travel direction (x) and chest wall-nipple direction (y).

\begin{tabular}{l|cc|cc} 
DBT system & $\begin{array}{c}\text { Frequency in tube travel direction [1/mm] } \\
\mathbf{M T F}_{\mathbf{m a x}}\end{array}$ & $\mathbf{M T F}_{\mathbf{5} 0}$ & $\begin{array}{c}\text { Frequency in chest wall-nipple direction [1/mm } \\
\mathbf{M T F}_{\mathbf{m a x}}\end{array}$ & $\mathbf{M T F}_{\mathbf{5}}$ \\
\hline Fujifilm HR & 0.6 & 1.3 & 1.9 & 4.1 \\
Fujifilm ST & 0.6 & 1.3 & 1.2 & 2.8 \\
GE & 0.3 & 1.3 & 1.4 & 3.5 \\
Hologic & 0.4 & 1.6 & 1.0 & 3.0 \\
Planmed & 0.3 & 1.4 & 0.5 & 1.5 \\
Siemens & 0.4 & 1.1 & 1.6 & 3.0
\end{tabular}

\subsection{Image noise}

Figure 5 shows the two-dimensional noise power spectra for the DBT systems. These are unique for all the studied systems. Figure 6 presents the corresponding relative NPS curves in the tube travel direction and chest wall-nipple direction. Hologic, GE and Planmed demonstrated similar noise textures between the directions whereas Fujifilm system showed the largest directional dependence as can be seen in the relative NPS curve shapes. Fujifilm HR deviated from the ST mode especially at higher frequencies in the tube travel direction. 


\subsection{Geometric accuracy}

Measured distances in the tube travel and chest wall-nipple directions are shown in table 4. The mean distances were mostly within $0.2 \mathrm{~mm}$ from the ground truth with the exceptions of Siemens and Hologic. In the Siemens DBT system, the reproduced distances were within $0.1 \mathrm{~mm}$ in the tube travel and within $0.5 \mathrm{~mm}$ in the chest wallnipple direction. Conversely, in the Hologic system, the measured distances were within $0.4 \mathrm{~mm}$ from the ground truth in the tube travel and within $0.2 \mathrm{~mm}$ in the chest wall-nipple direction. The clinical significance of these accuracies was not evaluated.

Table 4 - Measured distances ( \pm error) in the tube travel and chest wall-nipple directions. Propagation of uncertainty was used to estimate error. Measurement accuracy was \pm 1 pixel and pixel size was assumed to be accurate. The true lengths were $120 \mathrm{~mm}$ and $100 \mathrm{~mm}$, respectively.

\begin{tabular}{l|ccc|ccc} 
& \multicolumn{3}{|c}{$\begin{array}{c}\text { Measured distances in tube travel direction } \\
{[\mathbf{m m}]}\end{array}$} & \multicolumn{2}{c}{ Measured distances in chest wall-nipple direction } \\
DBT system & Bottom & Center & Top & Bottom & Center & Top \\
& & & & & & \\
\hline Fujifilm HR & $120.1 \pm 0.1$ & $119.8 \pm 0.1$ & $120.0 \pm 0.1$ & $100.1 \pm 0.1$ & $99.9 \pm 0.1$ & $100.0 \pm 0.1$ \\
Fujifilm ST & $120.1 \pm 0.2$ & $120.2 \pm 0.2$ & $120.1 \pm 0.2$ & $100.1 \pm 0.2$ & $100.2 \pm 0.2$ & $100.2 \pm 0.2$ \\
GE & $120.0 \pm 0.1$ & $119.9 \pm 0.1$ & $120.0 \pm 0.1$ & $100.0 \pm 0.1$ & $99.9 \pm 0.1$ & $99.9 \pm 0.1$ \\
Hologic & $120.3 \pm 0.2$ & $120.2 \pm 0.2$ & $120.4 \pm 0.1$ & $100.1 \pm 0.2$ & $100.1 \pm 0.2$ & $100.2 \pm 0.1$ \\
Planmed & $120.1 \pm 0.1$ & $120.0 \pm 0.1$ & $120.1 \pm 0.1$ & $99.9 \pm 0.1$ & $99.8 \pm 0.1$ & $100.0 \pm 0.1$ \\
Siemens & $119.9 \pm 0.1$ & $120.1 \pm 0.1$ & $119.9 \pm 0.1$ & $100.3 \pm 0.1$ & $100.4 \pm 0.1$ & $100.5 \pm 0.1$
\end{tabular}

\section{DISCUSSION}

In this study, we compared the technical image quality of five DBT systems. The added value of DBT in breast cancer screening has been shown in several publications. Large clinical trials employing Selenia Dimension DBT equipment have been reported: OTST trial ( $n=12621$ patients [2-3]); Storm trial ( $n=7292$ patients [4]); Rose et al. $(n=9499$ patients [7]); Haas et al. $(n=6100$ patients [8]); and Friedewald et al. $(n=173663$ patients [5]). The Malmö Breast tomosynthesis screening trial $(n=7500$ patients $)$ was done with a Siemens Mammomat Inspiration DBT system [6]. Inter-comparison between different manufacturers' DBT units has been limited. The proper use of DBT is also under debate. Furthermore, it is unclear whether the acquisition technique, scanning geometry, and reconstruction method affect lesion detectability. According to the current knowledge, DBT should be used in adjunct to two-dimensional mammography. Our phantom study with five DBT systems aimed to investigate 
whether there are differences in the technical image quality between the systems. One vendor had two scanning options for DBT imaging, and thus, we compared altogether six different setups.

Previously, a few publications have investigated the performance of different DBT systems [17-23]. These studies have compared, for example, the detector performance, automatic exposure control (AEC) performance, and image quality. Strudley et al. [17-19] compared the performance of Siemens Mammomat Inspiration and Hologic Selenia Dimensions DBT systems. Additionally, Strudley et al. [20] examined the performance of GE SenoClaire in a similar manner. Moreover, Rodríguez-Ruiz et al. [21] examined the performance of three different systems (Siemens Mammomat Inspiration, Hologic Selenia Dimensions, and Fujifilm Amulet Innovality), whereas in a study by Maldera et al. [22] four different DBT systems (Siemens Mammomat Inspiration, Hologic Selenia Dimensions, Fujifilm Amulet Innovality, and GE Senographe Essential) were investigated. Complementing the previous studies, we included Planmed Clarity in the comparisons. Similar to the current study, the aforementioned studies focused on technical imaging parameters and none of the studies investigated how a human observer can detect different objects in DBT images. Comparing previously published results may also be difficult as, for example, the software versions and reconstruction algorithms may vary. For example, in a study of Rodríguez-Ruiz et al. [21], the quality control mode was used for the Fujifilm system, resulting in a different pixel binning compared to our study. The reconstructed pixel size in their study was $100 \mu \mathrm{m} / 50 \mu \mathrm{m}$ for the ST and HR modes whereas $150 \mu \mathrm{m} / 100 \mu \mathrm{m}$ was used in our study due to the clinical operation modes used in our investigations. Additionally, the phantoms and analyzing method implementations may differ from study to study.

We found noticeable differences in technical image quality between the DBT systems. The ASF curve shapes vary considerably between the systems. The smallest ASF-FWHM values were found in the Fujifilm HR system and the highest in the Planmed DBT system. The ASF-FWHM values also varied in the Planmed system more than the other DBT systems. The x-ray tube rotation range seemingly affected the ASF-FWHM values: in most cases, the ASF-FWHM decreased when increasing the angular range of x-ray tube rotation. This supports the findings made by Rodríguez-Ruiz et al. [21] and Hu et al. [27]. However, the Planmed DBT system resulted in higher ASFFWHM values compared to Hologic and Fujifilm ST systems, although the Planmed DBT system used a higher angular range for x-ray tube rotation. We assume this to be related to different reconstruction methods and differences in the acquisition processes. The Planmed system uses a continuous sync-and-shoot image acquisition method, where the detector (and the breast or the phantom) rotates during the projection image acquisitions and 
always returns to its home position between the projections. Planmed also uses an iterative reconstruction algorithm. Furthermore, we also found lower ASF-FWHM values in the Fujifilm HR system compared to the Siemens system even though the angular range of the x-ray tube rotation was smaller in the Fujifilm HR. Moreover, the ASF-FWHM values seemed to increase in most of the DBT systems when the bead depths in the PMMA phantom were reduced in the z-direction. Exceptions to this rule were found in Planmed and Fujifilm HR systems. The ASFFWHM differences between the bead depths are mostly in accordance with Rodríguez-Ruiz et al. [21]. Moreover, when comparing our ASF measurement results in the mid-top bead position to those of Rodríguez-Ruiz et al. [21], the mean ASF-FWHM values measured in the Siemens system were $1.0 \mathrm{~mm}$ higher than in their measurements. In our study, we measured ASF-FWHM values to be $2.0 \mathrm{~mm}$ and $0.5 \mathrm{~mm}$ smaller than they measured for Fujifilm DBT system using ST-mode and HR-mode, respectively. In the case of the Hologic system, our measurements yielded similar results to Rodríguez-Ruiz et al. [21]

Similar to ASF measurements, we also noticed differences in in-plane resolutions between the systems in our MTF measurements performed both in tube travel direction and chest wall-nipple direction. The image resolution was particularly higher in the chest wall-nipple direction compared to tube travel direction in each equipment except the Planmed DBT system. The Planmed system resulted in the most comparable MTF values between the two directions. The variation in MTF values between the DBT systems was notably smaller in tube travel direction compared to chest wall-nipple direction. Compared to the Rodríguez-Ruiz et al. [21] study, we observed lower (0.6 to $\left.0.0 \mathrm{~mm}^{-1}\right) \mathrm{MTF}_{50}$ values in the tube travel direction, with $25 \mu \mathrm{m}$ tungsten wire for the Siemens, Hologic, and Fujifilm systems. However, we measured slightly higher $\left(0.2\right.$ to $\left.0.4 \mathrm{~mm}^{-1}\right) \mathrm{MTF}_{50}$ values in the chest wall-nipple direction compared to their study, except in the case of the Siemens system, with which we measured lower $(-0.3$ $\left.\mathrm{mm}^{-1}\right) \mathrm{MTF}_{50}$ values.

Our image-noise analysis showed clear differences between the studied DBT systems. Both resolution and noise were directionally dependent, but the extent of anisotropicity was unique to each system. This may lead to vastly differing clinical image textures but also differences in malignancy conspicuity. The low noise power in the $\mathrm{x}$-axis may result from ramp-like filters applied to the projection images [26]. The Planmed system NPS curves differed from the other systems probably due to different image reconstruction algorithms and reconstructed image pixel size. The Planmed system also had highly isotropic resolution (see the MTF curves in Figure 4) when compared to the others, and the noise can be seen at these same (in the y-direction relatively low) frequencies. This causes the 
image noise to appear larger (“grainier”). Geometric accuracy was good overall, although small deviations were found in the Siemens and Hologic systems.

Our study has certain limitations. First and foremost, the phantom images are never a complete substitute for patient acquisitions. Metallic high-contrast targets, both beads and wires, have greater attenuation than the microcalcifications seen in real breast tissue. We also failed to assess the low-contrast detectability in our study. Noise and resolution may have various non-linear contrast and dose dependencies due to differences in the reconstruction algorithms. In addition, we did not harmonize the dose levels between the systems, but instead used an automatic exposure control in our measurements. This choice may introduce limitations to the inter-device comparability. The rapid development of the imaging techniques and reconstruction algorithms (also in the form of software updates to existing setups) complicates the definite comparison between the systems.

\section{CONCLUSION}

This phantom study evaluated the technical image quality of five digital breast tomosynthesis devices. The systems have different technical and data processing solutions, leading to notable differences in the obtained results. The in-plane resolution was higher in the chest wall-nipple direction than in the tube travel direction in all the systems. The ASF-FWHM values varied remarkably between the systems, for which the angular range of the system seemed to be a contributing factor. A wider angular range tended to reduce artefact spreading in the z-direction. Furthermore, differences in the noise properties of the devices were observed. The geometric accuracy of all the systems was within $0.5 \%$.

\section{ACKNOWLEDGEMENTS}

The authors are grateful to Planmed Oy for borrowing phantoms.

Funding: This study was supported by Helsinki University Hospital research funding. 


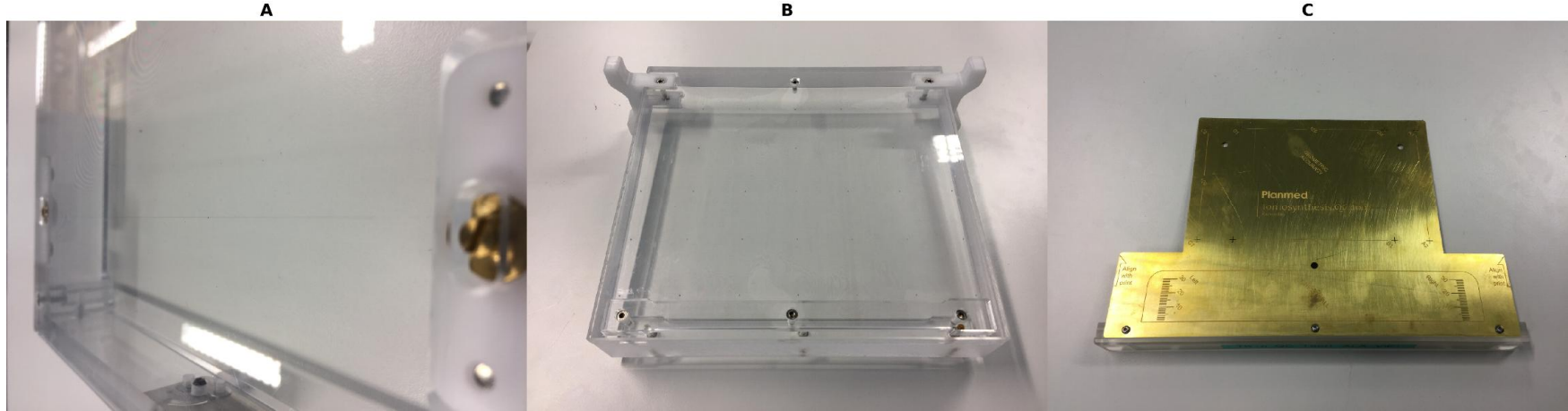

Figure 1 - Image quality phantoms that were used in this study: A) a wire phantom was used to measure MTF, B) a bead phantom was used to measure artefact spread function, and C) a geometric accuracy phantom was used together with PMMA plates to determine the geometric accuracy at different heights.

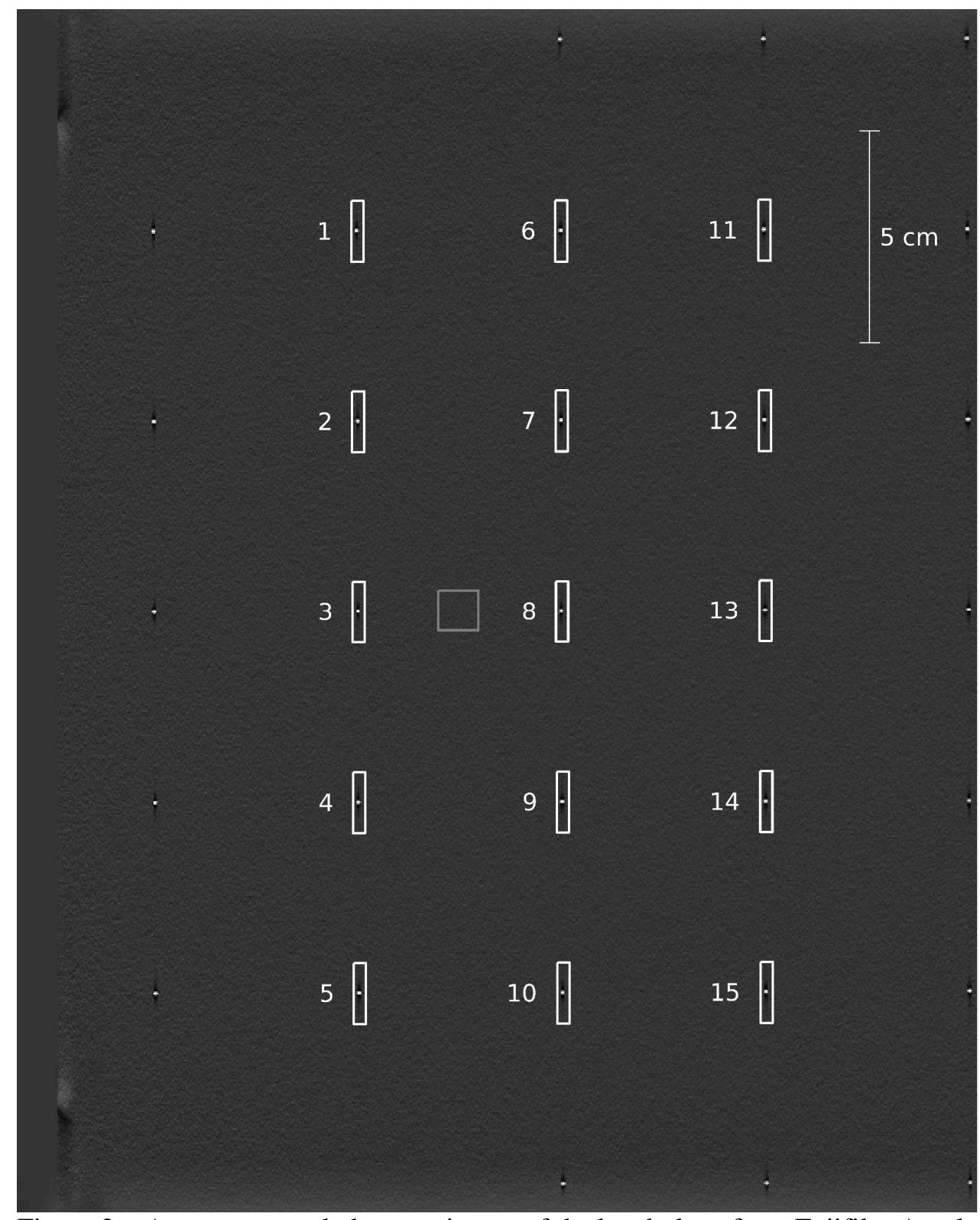

Figure 2 - A reconstructed phantom image of the bead plane from Fujifilm Amulet Innovality (HR mode) system. Bead numbers 1-15 were used in the comparison. A grey square indicates the background area and white squares the maximum artefact search areas used to calculate the ASF. The $5 \mathrm{~cm}$ long line in top right corner is to give a scale to image. 

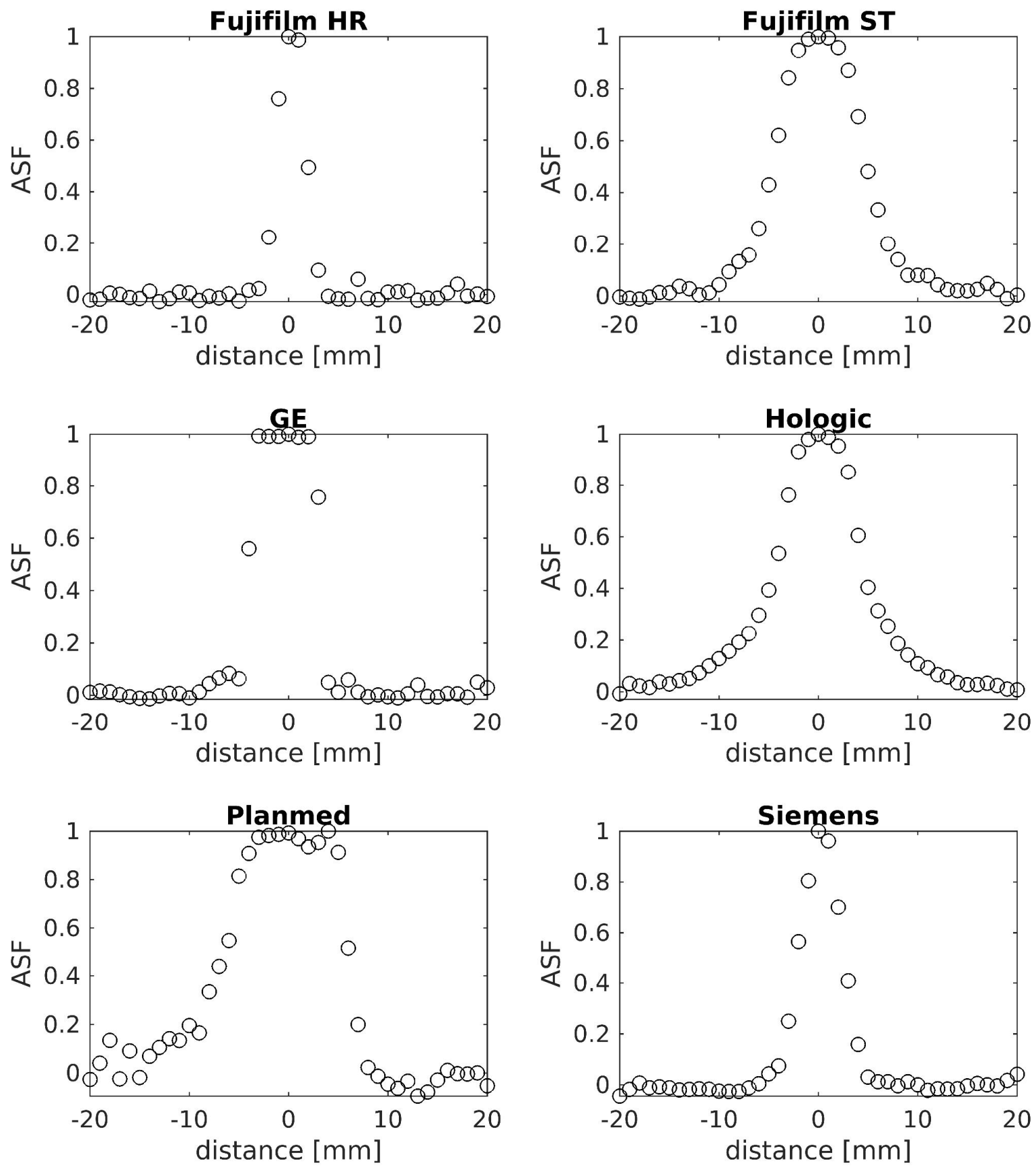

Figure 3 - Shapes of the ASF curves measured from the bead number three in the mid-top position. 

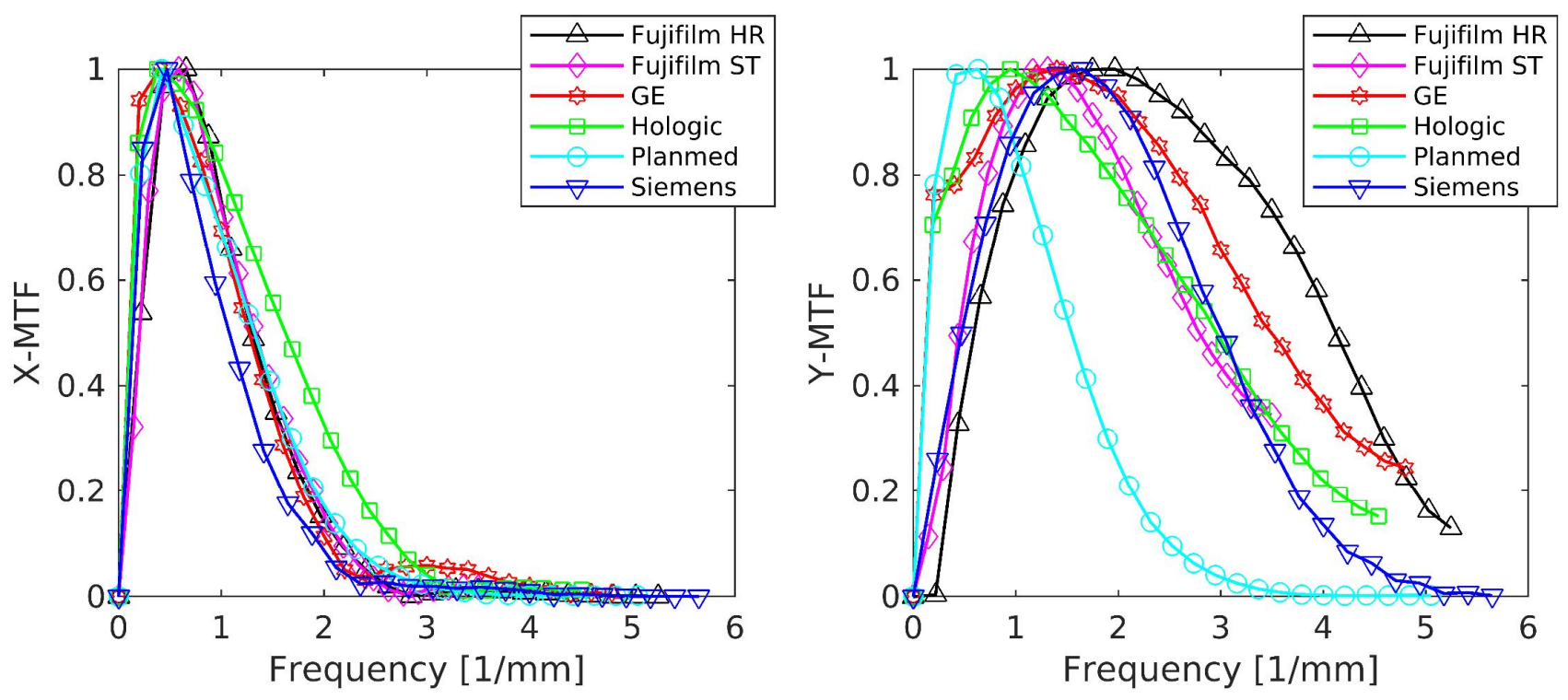

Figure 4 - MTF curves in the tube travel direction (left) and in the chest wall-nipple direction (right).
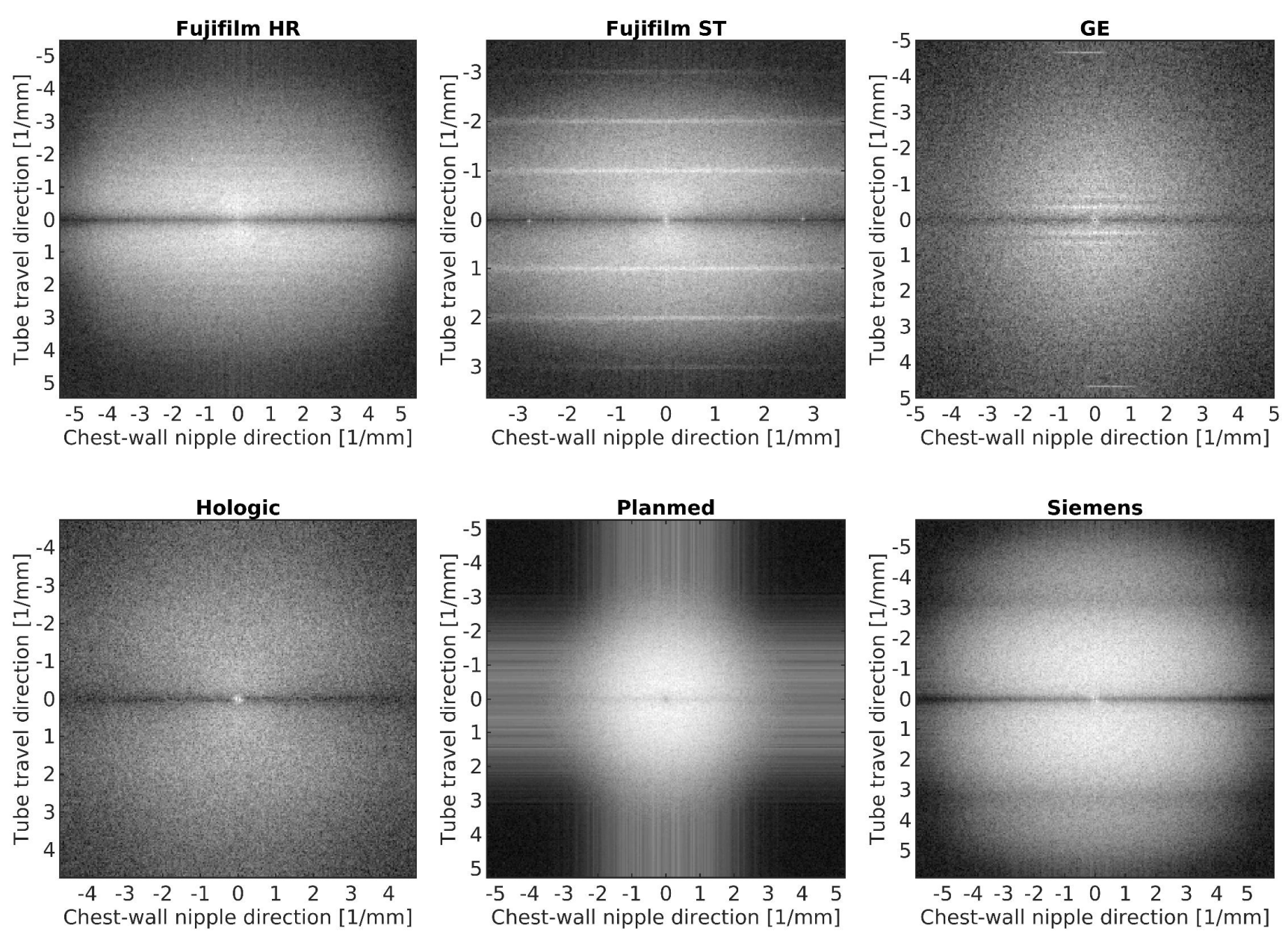

Figure 5 - The logarithm of two-dimensional noise power spectra, calculated from the center area of the reconstructed plane, are distinctly unique for all the studied DBT systems. 

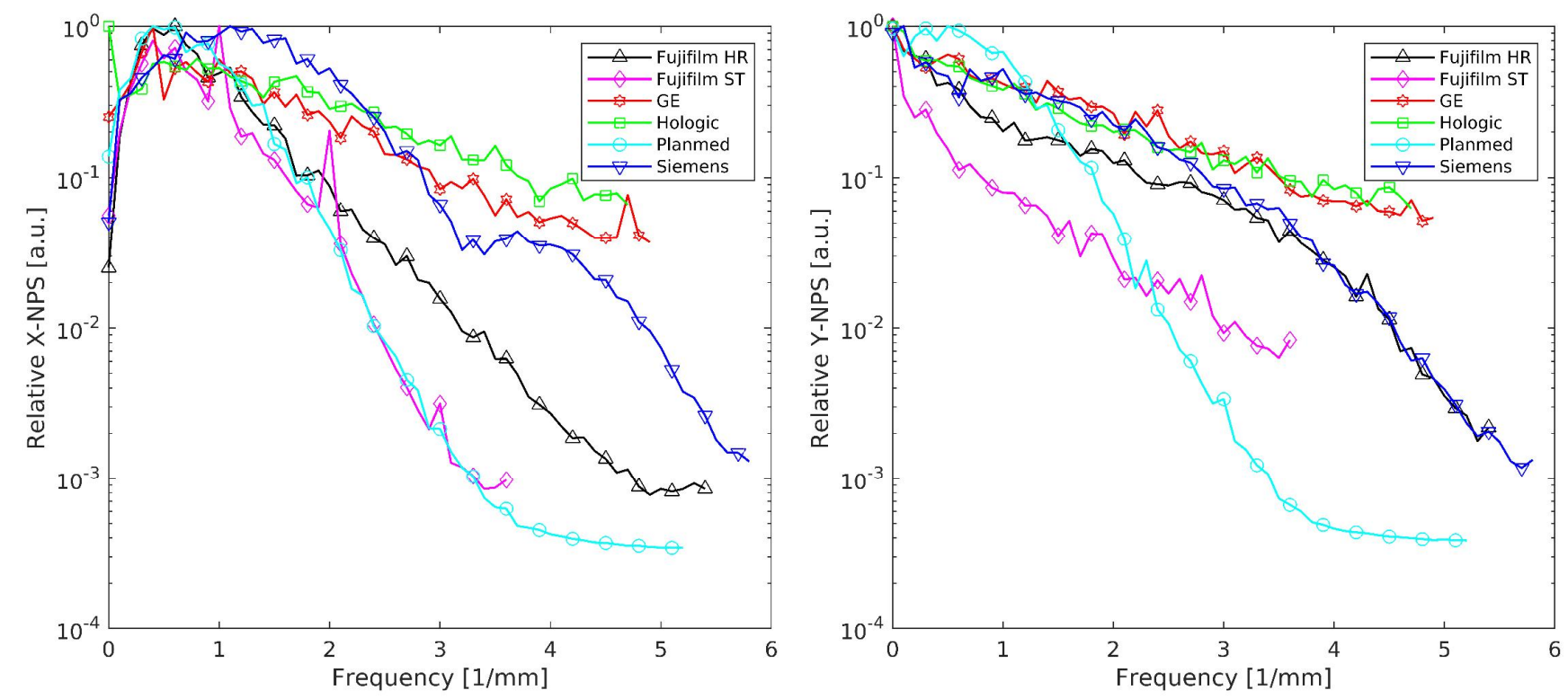

Figure 6 - Relative NPS curves in the tube travel direction (left) and in the chest wall-nipple direction (right).

\section{REFERENCES}

[1] Clark CR, Tosteson TD, Tosteson ANA, Onega T, Weiss JE, Harris KA, et al. Diffusion of digital breast tomosynthesis among women in primary care: associations with insurance type, Cancer Medicine 2017; 6(5):1102-1107

[2] Skaane P, Bandos AI, Gullien R, Eben EB, Ekseth U, Haakenaasen U, et al. Prospective trial comparing full-field digital mammography (FFDM) versus combined FFDM and tomosynthesis in a populationbased screening programme using independent double reading with arbitration. Eur Radiol 2013;23:206171.

[3] Skaane P, Bandos AI, Gullien R, Eben EB, Ekseth U, Haakenaasen U, et al. Comparison of digital mammography alone and digital mammography plus tomosynthesis in a population-based screening program. Radiology 2013;267:47-56.

[4] Ciatto S, Houssami N, Bernardi D, Caumo F, Pellegrini M, Brunelli S, et al. Integration of 3D digital mammography with tomosynthesis for population breast-cancer screening (STORM): A prospective comparison study. Lancet Oncol 2013;14:583-9.

[5] Friedewald SM, Rafferty EA, Rose SL, Durand MA, Plecha DM, Greenberg JS, et al. Breast Cancer Screening Using Tomosynthesis in Combination With Digital Mammography. Jama 2014;311:2499.

[6] Lång K, Andersson I, Rosso A, Tingberg A, Timberg P, Zackrisson S. Performance of one-view breast tomosynthesis as a stand-alone breast cancer screening modality: results from the Malmö Breast 
Tomosynthesis Screening Trial, a population-based study. Eur Radiol 2016;26:184-90.

[7] Rose SL, Tidwell AL, Bujnoch LJ, Kushvaha AC, Nordmann AS, Sexton R. Implementation of breast tomosynthesis in a routine screening practice: An observational study. Am J Roentgenol 2013;200:14018.

[8] Haas BM, Kalra V, Geisel J, Raghu M, Durand M, Philpotts LE. Comparison of tomosynthesis plus digital mammography and digital mammography alone for breast cancer screening. Radiology 2013;269:694-700.

[9] Spangler ML, Zuley ML, Sumkin JH, Abrams G, Ganott MA, Hakim C, et al. Detection and classification of calcifications on digital breast tomosynthesis and 2D digital mammography: A comparison. Am J Roentgenol 2011;196:320-4.

[10] Tagliafico A, Mariscotti G, Durando M, Stevanin C, Tagliafico G, Martino L, et al. Characterisation of microcalcification clusters on 2D digital mammography (FFDM) and digital breast tomosynthesis (DBT): does DBT underestimate microcalcification clusters? Results of a multicentre study. Eur Radiol 2014;25:9-14.

[11] Kopans D, Gavenonis S, Halpern E, Moore R. Calcifications in the breast and digital breast tomosynthesis. Breast J 2011;17:638-44.

[12] Michell MJ, Iqbal A, Wasan RK, Evans DR, Peacock C, Lawinski, et al. A comparison of the accuracy of film-screen mammography, full-field digital mammography, and digitalbreast tomosynthesis. Clin Radiol 2012;67:976-81.

[13] Poplack SP, Tosteson TD, Kogel CA, Nagy HM. Digital breast tomosynthesis:initial experience in 98 women with abnormal digital screeningmammography. AJR Am J Roentgenol 2007;189:616-23.

[14] Gilbert FJ, Tucker L, Young KC. Digital breast tomosynthesis (DBT): a review of the evidence for use as a screening tool. Clin Radiol 2016;71:141-150.

[15] Sechopoulos I. A review of breast tomosynthesis. Part I. The image acquisition process. Med Phys 2013;40(1):014301.

[16] EUREF, Protocol for the Quality Control of the Physical and Technical Aspects of Digital Breast Tomosynthesis Systems version 1.01, 2016.

[17] Strudley CJ, Looney P, Young KC. Technical evaluation of Hologic Selenia Dimensions digital breast tomosynthesis system v2, National Health Services Breast Screening Program Equipment Report 1307, 2014. 
[18] Strudley CJ, Warren LM, Young KC. Technical evaluation of Siemens Mammomat Inspiration digital breast tomosynthesis system v2. National Health Services Breast Screening Program Equipment Report 1306, 2015.

[19] Strudley CJ, Young KC, Looney P, Gilbert FJ. Development and experience of quality control methods for digital breast tomosynthesis systems. Br J Radiol 2015;88:20150324.

[20] Strudley CJ, Oduko JM, Young KC. Technical evaluation of GE Healthcare SenoClaire digital breast tomosynthesis system, National Health Services Breast Screening Program Equipment Report 1404, 2016.

[21] Rodríguez-Ruiz A, Castillo M, Garayoa J, Chevalier M. Evaluation of the technical performance of three different commercial digital breast tomosynthesis systems in the clinical environment. Phys Med 2016;32:767-77.

[22] Maldera A, De Marco P, Colombo PE, Origgi D, Torresin A, Digital breast tomosynthesis: Dose and image quality assessment, Physica Medica 2017;33:56-67.

[23] Marshall NW, Bosmans H. Measurements of system sharpness for two digital breast tomosynthesis systems. Phys Med Biol 2012;57:7629-50.

[24] Mainprize JG, Bloomquist A, Wang X, Martin JY. Dependence of image quality on geometric factors in breast tomosynthesis. Med Phys 2011;38:3090-103.

[25] https://www.efomp.org/ (accessed 17th April 2019).

[26] Wu T, Moore RH, Rafferty EA, Kopans DB. A comparison of reconstruction algorithms for breast tomosynthesis. Med Phys 2004;31:2636-47.

[27] Hu YH, Zhao B, Zhao W. Image artifacts in digital breast tomosynthesis: Investigation of the effects of system geometry and reconstruction parameters using a linear system approach. Med Phys 2008;35(12):5242-52. 
Appendix 1: Mean glandular doses in the phantom measurements

Table 5 - Mean glandular doses in the MTF measurements.

\begin{tabular}{l|cc} 
DBT system & \multicolumn{2}{|c}{ Mean glandular dose } \\
& Y-MTF [mGy] & X-MTF [mGy] \\
\hline Fujifilm HR & 3.34 & 3.34 \\
Fujifilm ST & 3.35 & 3.35 \\
GE & 0.76 & 0.76 \\
Hologic & 1.50 & 1.47 \\
Planmed & 1.61 & 1.61 \\
Siemens & 0.63 & 0.62
\end{tabular}

Table 6 - Mean glandular doses in the ASF measurements.

\begin{tabular}{l|cccc} 
& \multicolumn{4}{|c}{ Mean glandular dose } \\
DBT system & Bottom (12 $\mathbf{~ m m})$ & Mid-bottom $(\mathbf{2 4} \mathbf{~ m m})$ & Mid-top (36 mm) & Top (48 mm) \\
& {$[\mathbf{m G y}]$} & {$[\mathbf{m G y}]$} & {$[\mathbf{m G y}]$} & {$[\mathbf{m G y}]$} \\
\hline Fujifilm HR & 3.47 & 3.35 & 3.35 & 3.47 \\
Fujifilm ST & 2.07 & 2.07 & 2.07 & 2.27 \\
GE & 1.76 & 1.74 & 1.70 & 1.74 \\
Hologic & 2.00 & 1.95 & 2.00 & 1.96 \\
Planmed & 1.84 & 1.84 & 1.89 & 1.88 \\
Siemens & 2.12 & 2.07 & 2.07 & 2.12
\end{tabular}

Table 7 - Mean glandular doses in the geometric accuracy measurements.

\begin{tabular}{l|ccc} 
& \multicolumn{3}{|c}{ Mean glandular dose } \\
DBT system & Bottom [mGy] & Center [mGy] & Top [mGy] \\
\hline Fujifilm HR & 2.33 & 2.33 & 2.33 \\
Fujifilm ST & 2.33 & 2.33 & 2.33 \\
GE & 2.38 & 2.38 & 2.41 \\
Hologic & 3.11 & 3.08 & 3.11 \\
Planmed & 1.94 & 2.26 & 1.95 \\
Siemens & 2.21 & 2.21 & 2.21
\end{tabular}

